\title{
MicroRNA-106a regulates the proliferation and invasion of human osteosarcoma cells by targeting VNN2
}

\author{
YAO CHEN, TAO HUANG, XIAOFAN YANG, CHAOWU LIU, PENGCHENG LI, \\ ZHIHANG WANG and SHENGPENG ZHI \\ Department of Orthopedics, The First Affiliated Hospital of China Medical University, \\ Shenyang, Liaoning 110001, P.R. China
}

Received November 9, 2017; Accepted July 17, 2018

DOI: $10.3892 /$ or.2018.6601

\begin{abstract}
MicroRNAs (miRs) serve an essential role in tumorigenesis and are able to act as tumor suppressor genes or oncogenes. miR-106a has been identified generally as an oncogene in multiple types of human cancer; however, its association with osteosarcoma has not previously been understood. Reverse transcription-quantitative polymerase chain reaction (RT-qPCR) was used to detect miR-106a expression in 18 osteosarcoma tissues compared with paired non-cancerous adjacent tissues as well as osteosarcoma cell lines (U2OS, Saos-2 and MG63) compared with a normal osteoblast cell line (hFOB1.19). The biological function of U2OS cells was assessed by using a Transwell cell invasion assay, MTS proliferation assay and flow cytometric analysis following the transfection with lentivirus-mediated small interfering RNA (miR-106a-inhibitor). Western blotting and Luciferase reporters were used to investigate whether VNN2 was a target of miR-106a in osteosarcoma cells. Based on the RT-qPCR data, miR-106a was significantly upregulated in osteosarcoma tissues and osteosarcoma cell lines compared with their control counterparts $(\mathrm{P}<0.01)$. The knockdown of miR-106a resulted in cell proliferation and invasion inhibition. Furthermore, apoptosis enhancement and G2/M cell cycle arrest were detected by flow cytometry. The western blot analysis indicated that U2OS cells infected with miR-106a-inhibitor lentivirus had a higher VNN2 protein expression level compared with cells infected with miR-106a-negative control lentivirus. Luciferase reporters containing the 3'-untranslated region sequence of VNN2 messenger RNA demonstrated VNN2 may be a target of miR-106a. In addition, a negative correlation was confirmed between the expression of VNN2 and miR-106a in the tumor samples. The results of the present study indicate that the knockdown of miR-106a overexpressed
\end{abstract}

Correspondence to: Professor Tao Huang, Department of Orthopedics, The First Affiliated Hospital of China Medical University, Shenyang, Liaoning 110001, P.R. China

E-mail: huangtao_cmu@126.com

Key words: miR-106a, VNN2, osteosarcoma, U2OS cells
VNN2 to inhibiting the proliferation, migration and invasion as well as inducing the apoptosis of human osteosarcoma cells.

\section{Introduction}

Osteosarcoma is one of the most common types of serious malignant bone tumor with high mortality rates in children and adolescents $(1,2)$. With neoadjuvant chemotherapy and surgery widely used in clinical treatment, the 5-year survival rate of osteosarcoma is able to reach $50-60 \%$ (3). However, the survival rate remains $30 \%$ in patients with distant metastatic tumors and $\sim 60 \%$ of patients with osteosarcoma are diagnosed with small metastases, which suggests a poor prognosis $(4,5)$. Therefore, it is important to identify the molecular mechanism underlying osteosarcoma invasion and metastasis to explore novel therapeutic targets of osteosarcoma.

MicroRNAs (miRNAs) are endogenous small noncoding RNAs beTween-20 and 24 nucleotides in length that bind to the 3'untranslated region (UTR) of target mRNAs to silence target gene expression (6). miRNAs are post-transcriptional regulators, serving important roles in a variety of physiological and pathological processes, including morphogenesis, differentiation and carcinogenesis (7-9). In a previous study, various miRNAs have been demonstrated to be dysregulated in multiple types of cancer, including osteosarcoma (10). Certain studies have also highlighted the association between miRNAs, and abnormal regulation of proliferation, invasion, apoptosis and cell cycle distribution (11-13). Certain miRNAs have been identified to be dysregulated in osteosarcoma (14); however, to the best of our knowledge, the role of miR-106a has not previously been investigated.

Vascular non-inflammatory molecule $2(\mathrm{VNN} 2)$ protein is a novel glycosylphosphatidyl inositol-anchored protein member of the VNN family that serves an important role in transendothelial migration of cells (15). The VNN family includes membrane-associated proteins, a few of which have been reported to participate in regulating neutrophil trafficking and adherence (16). It is generally accepted that neutrophils are present in numerous different types of cancer and are eventually recruited to the tumor microenvironment (17). Infiltrating inflammatory cells are highly prevalent within the tumor microenvironment and mediate numerous processes involved in tumor progression in vivo (18). In addition, the VNN family 
belongs to a wider pantetheinase family and serve a role in redox regulation, which may associate with tumor progression in vitro (19). Given that miR-106a is upregulated in human osteosarcoma cells, and associated with cell proliferation, invasion and apoptosis, we hypothesized that the knockdown of miR-106a may result in VNN2 gene overexpression. The present study aimed to determine the expression and function of miR-106a in human U2OS cell lines and tumor tissues obtained from patients with osteosarcoma. The results revealed that the knockdown of miR-106a mediated tumor progression at least partially by targeting VNN2 in osteosarcoma.

\section{Materials and methods}

Patients and tissue samples. Osteosarcoma tissues and matched non-cancerous adjacent tissues (NATs) were obtained from 18 patients ( 9 males and 9 females; mean age, 22.7 years; age range, 15-35 years) histopathologically diagnosed with osteosarcoma at the First Affiliated Hospital of China Medical University (Shenyang, China). No patients in the study received any preoperative treatment, and all patients underwent resection of osteosarcoma at the time of diagnosis between June 2013 and June 2016. Patients with histological grade IIB/III osteosarcoma were included, and patients with any other primary disease were excluded. The paired and osteosarcoma tissues were immediately preserved in liquid nitrogen and maintained at $-80^{\circ} \mathrm{C}$. All associated clinical data, including age, sex and tumor node metastasis stage were obtained from the medical records of patients. The present study received approval by the Ethics Committee of the First Affiliated Hospital of China Medical University and written informed consent was obtained from all patients.

Cell lines and culture. Human osteosarcoma U2OS (cat. no. TCHu88), Saos-2 (cat. no. TCHu114), MG63 (cat. no. TCHu124), 293T (cat. no. GNHu17) and human osteoblast hFOB1.19 (cat. no. GNHu14) cells were purchased from the Institute of Biochemistry and Cell Research Center at the Chinese Academy of Sciences (Shanghai, China). The U2OS, Saos-2 and MG63 cells were cultured in high-glucose Dulbecco's modified Eagle medium (DMEM) containing 10\% fetal bovine serum (both from HyClone; GE Healthcare Life Sciences, Logan City, UT, USA) and hFOB1.19 cells were cultured in DMEM/F12 medium (HyClone; GE Healthcare Life Sciences) containing 10\% fetal bovine serum. All cells were cultured at $37^{\circ} \mathrm{C}$ in a humidified atmosphere with $5 \%$ $\mathrm{CO}_{2}$.

Lentivirus infection and plasmid transfection. miR-106a gene-expression plasmid (miR-106a), miR-106a gene-inhibition plasmid packaged into letivirus core vector (hU6-MCS-Ubiquitin-EGFP-IRES-puromycin) (miR-106a-inhibitor), mock negative control (miR-NC), VNN2 wild type gene over-expression plasmid (GV272/VNN2), VNN2 mutant type gene over-expression plasmid (GV272/VNN2-mut), VNN2 negative control plasmid (GV272/VNN2-NC) were all chemically synthesized by Shanghai GeneChem Co., Ltd. (Shanghai, China). miR-106a-inhibitor was used as the experimental group and miR-NC as the control group. The experimental group and control group were treated with lentivirus, which was inactivated and served as a carrier. Therefore, the blank control group was removed (20). The sequences were as follows: miR-106a-inhibitor, 5'-GTAAGAAGTGCTTACATTGCAG-3'; miR-NC, 5'-TTCTCCGAACGTGTCACGT-3'; and miR-106a, 5'-ACG GGCCCTCTAGACTCGAGTGTTTTAACCAGGTGAGTC-3'. The sequence of miR-106a binding site in GV272/VNN2 3'UTR was 5'-GCCATTGCAAA-3', and GV272/VNN2-mut 3'UTR was 5'-GCACGGTACAA-3'. All plasmid DNA was extracted using an EndoFree Mini Plasmid kit (Tiangen Biotech Co., Ltd., Beijing, China). U2OS cells were transfected with the plasmid using X-tremeGENE HP (Roche Diagnostics, Basel, Switzerland) and infected at a multiplicity of infection of 10 for $12 \mathrm{~h}$ in the incubator, then incubated for $72 \mathrm{~h}$ to perform the subsequent trials.

$R N A$ extraction and reverse transcription-quantitative polymerase chain reaction $(R T-q P C R)$. Total RNA from the paired specimens, and U2OS, Saos-2, MG63, human hFOB1.19 cells and infected U2OS cells were isolated using the QIAzol reagent (Qiagen, Inc., Valencia, CA, USA) according to the manufacturer's protocol. The stem-loop reverse-transcription primers were designed as follows: miR-106a, 5'-GTCGTA TCCAGTGCAGGGTCCGAGGTATTCGCACTGGATACG ACCTACCT-3' and U6 small nuclear RNA (U6), 5'-CTC AACTGGTGTCGTGGAGTCGGCAATTCAGTTGAGGGG ACAAA-3'. The oligo dT primers were used for VNN2 and GAPDH genes. Reverse transcription was performed using a PrimeScript RT reagent kit (Takara Bio, Inc., Otsu, Japan) according to the manufacturer's protocol. The sequences of the PCR primers were designed as follows: miR-106a forward, 5'-CGCAAAAGTGCTTACAGTGCA-3' and reverse, 5'-GTG CAGGGTCCGAGGT-3'; U6 forward, 5'-CTCGCTTCG GCAGCACA-3' and reverse, 5'-AACGCTTCACGAATT TGCGT-3'; VNN2 forward, 5'-CCATAAGGTGGGCAA GAGTCA-3' and reverse, 5'-CTCCGGCTTTTCAGGGAC AT-3'; GAPDH forward, 5'-GCACCGTCAAGGCTGAGA AC-3' and reverse, 5'-TGGTGAAGACGCCAGTGGA-3'. All PCR reactions were performed using an Applied Biosystems 7900HT Real-Time PCR system (Applied Biosystems; Thermo Fisher Scientific, Inc., Waltham, MA, USA) using the SYBR PrimeScript RT-PCR kit (Takara Bio, Inc.) according to the manufacturer's protocol. The thermocycling conditions maintained were as follows: $95^{\circ} \mathrm{C}$ for $30 \mathrm{sec} ; 95^{\circ} \mathrm{C}$ for $5 \mathrm{sec}$; and $60^{\circ} \mathrm{C}$ for $32 \mathrm{sec}$, for 40 cycles. To ensure the fidelity of PCR reactions results, the expression levels of miR-106a were normalized to the expression of U6, while the expression levels of VNN2 were normalized to the expression of GAPDH. The relative expression levels were calculated and normalized using the $2^{-\Delta \Delta \mathrm{Cq}}$ method (21).

Cell proliferation assay. The proliferation ability of infected miR-106a-inhibitor and miR-106a-NC U2OS cells was measured using an MTS assay kit (Promega Corporation, Madison, WI, USA) according to the manufacturer's protocol. Cells were plated $\left(2 \times 10^{3}\right.$ cells/well) in 96-well plates, and cultured for 24, 48, 72 and $96 \mathrm{~h}$. In a 96-well plate, $20 \mu \mathrm{l}$ MTS reagent was added for every $100 \mu \mathrm{l}$ medium/well and then incubated at $37^{\circ} \mathrm{C}$ in $5 \% \mathrm{CO}_{2}$ for $4 \mathrm{~h}$. The optical density value of each sample was recorded at the wavelength of $490 \mathrm{~nm}$ via a microplate reader. 
Cell migration and invasion assays. The migration and invasion ability of infected U2OS cells were detected using Transwell assays. The upper and lower chambers of a 24-well plate (Corning Incorporated, Corning, NY, USA) were washed with serum-free DMEM, and then $40 \mu 1$ Matrigel (BD Biosciences, San Jose, CA, USA) diluted 1:8 with serum-free medium was added to the upper chamber to evenly cover the surface of the polycarbonate membrane for the invasion assay, whereas no Matrigel was added for migration assay. The 24-well plate was put into an incubator for $4 \mathrm{~h}$ at $37^{\circ} \mathrm{C}$ to allow the Matrigel to solidify. A total of $200 \mu 1$ DMEM containing $1 \times 10^{5}$ cells was seeded on the top of the upper chamber in the Transwell invasion chamber while $600 \mu \mathrm{l}$ DMEM with $10 \%$ FBS was added into the lower chamber. The Transwell invasion assay was put in a cell incubator at $37^{\circ} \mathrm{C}$ with $5 \% \mathrm{CO}_{2}$. After $48 \mathrm{~h}$, the cells on the upper surface were wiped away using a wet cotton swab. For the adherent cells, the migrated cells attach to the other side of the membrane in a Transwell assay (22). Cells that passed through the upper chamber and attach to the lower surface were fixed using absolute ethanol for $15 \mathrm{~min}$ at room temperature and stained with $0.1 \%$ crystal violet for $15 \mathrm{~min}$ at room temperature. Visible cells were counted using an inverted phase contrast microscope at a magnification of $x 400$.

Analysis of cell apoptosis. After infection for $72 \mathrm{~h}$, cells were detached using EDTA-free trypsin and washed three times with ice-cold PBS. The cell apoptosis rate was detected using the FITC Annexin V Apoptosis Detection kit (BD Biosciences) according to the manufacturer's protocol using a flow cytometer.

Analysis of cell cycle. Cells in the logarithmic phase were harvested following infection for $72 \mathrm{~h}$ and washed twice with ice-cold PBS, then fixed with $5 \mathrm{ml} 70 \%$ ethanol overnight at $-20^{\circ} \mathrm{C}$. Fixed cells were washed twice with ice-cold PBS and then centrifuged at $800 \mathrm{xg}$ for $15 \mathrm{~min}$ at $4^{\circ} \mathrm{C}$. Cells were resuspended in $0.4 \mathrm{ml}$ ice-cold PBS and subjected to $1 \mathrm{ml}$ propidium iodide (PI)/Triton X-100 containing RNase staining for $30 \mathrm{~min}$ at $4^{\circ} \mathrm{C}$ followed by flow cytometry. Data were analyzed using the CellQuest software (version 7.5.3; BD Biosciences).

Dual-luciferase reporter assay. Cells were seeded into a 24-well plate $\left(1 \times 10^{5}\right.$ cells/well) and co-transfected with $0.4 \mu \mathrm{g}$ of miR-106a or miR-NC vectors, and $0.1 \mu \mathrm{g}$ of GV272/VNN2 or GV272/VNN2-mut vectors containing 3'-UTR as well as GV272/VNN2-NC vector. The firefly luciferase reporter gene was constructed into the vectors to quantitatively reflect the inhibitory effect. Cells were harvested $48 \mathrm{~h}$ after transfection for luciferase activity assays using the Reporter Assay system (Promega Corporation) according to the manufacturer's protocol.

Western blot analysis. Cells were harvested $72 \mathrm{~h}$ after infection, and total protein was extracted using radio immunoprecipitation assay lysis buffer and phenylmethanesulfonyl fluoride (both from Beyotime Institute of Biotechnology, Shanghai, China) according to the manufacturer's protocol. Total protein $(30 \mu \mathrm{g} / \mathrm{lane})$ were separated using $10 \%$ SDS-PAGE and electroblotted onto a $0.2-\mu \mathrm{m}$ pore size polyvinylidene fluoride membrane (Beyotime Institute of Biotechnology). The
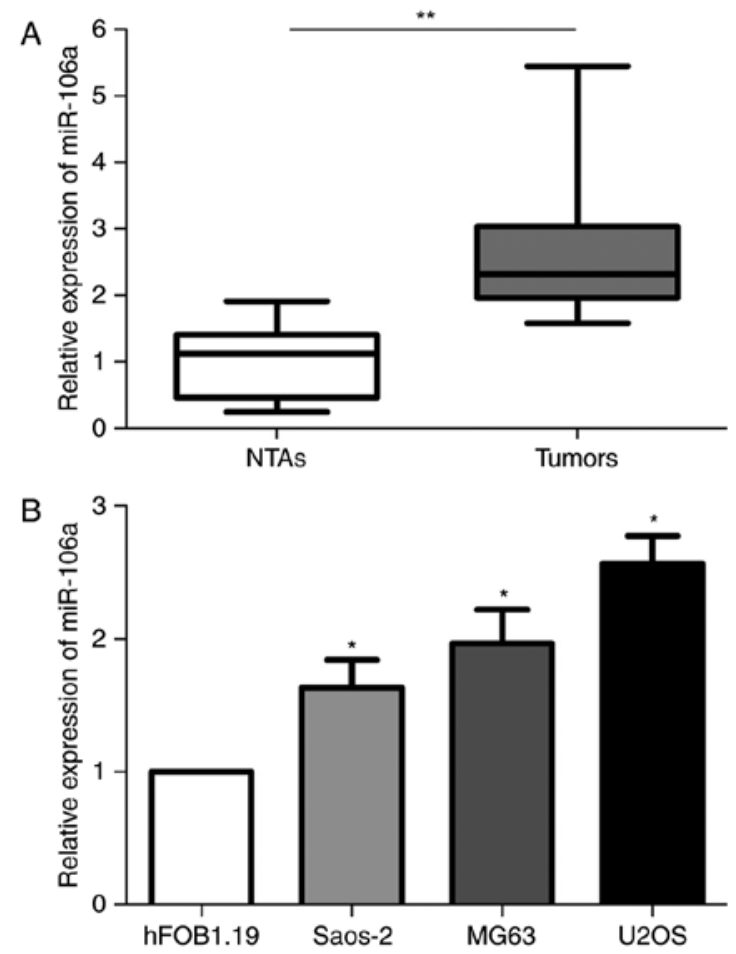

Figure 1. Relative expression of miR-106a in osteosarcoma tissues and three osteosarcoma cell lines. (A) Relative expression of miR-106a in 18 osteosarcoma samples compared with NTAs was determined using reverse transcription-quantitative polymerase chain reaction. (B) Expression levels of miR-106a in U2OS, Saos-2 and MG63 cells compared with normal osteoblast hFOB1.19 cells. ${ }^{*} \mathrm{P}<0.05$. Abundance of miR-106a was normalized to U6. Data are presented as the mean \pm standard deviation of three independent experiments. miR, microRNA; NTAs, non-cancerous adjacent tissues.

membrane was blocked by 5\% skim milk at room temperature for $2 \mathrm{~h}$ and then incubated overnight at $4^{\circ} \mathrm{C}$ with rabbit anti-human VNN2 antibody (1:1,000; cat. no., 25643-1-AP) or $\beta$-actin antibody (1:1,000; cat. no., 20536-1-AP) (both from ProteinTech Group, Inc., Chicago, IL, USA) as a control. Following washing of the membrane three times with Tris-buffered saline containing Tween-20 (1X TBST), the membrane was incubated for $2 \mathrm{~h}$ at room temperature with horseradish peroxidase-conjugated affinipure goat anti-rabbit IgG secondary antibody (1:5,000; cat. no., SA00001-2; ProteinTech Group, Inc.). Following washing of the membrane three times with TBST again, an ultrasensitive chemiluminescence solution was used from the BeyoECL Plus kit (Beyotime Biotechnology, Shanghai, China) to detect the protein bands according to the manufacturer's protocol (23). The bands were observed using MF-Chemisis 2.0 and GelCapture software (DNR Bio-Imaging Systems, Ltd., Neve Yamin, Israel).

Bioinformatic and statistical analysis. Open access miRNA databases (TargetScan, http://www.targetscan.org/vert_72/; PicTarget, https://pictar.mdc-berlin.de/; and MicroCosm, https://www.ebi.ac.uk/enright-srv/microcosm/) were used for prediction of miR-106a target genes. SPSS software (version 22; IBM Corp., Armonk, NY, USA) was used to analyze all results. All statistical data are presented as mean \pm standard deviation following three independent experiments. Student's t-tests were used when only two groups were present and one-way analysis of variance followed by the 


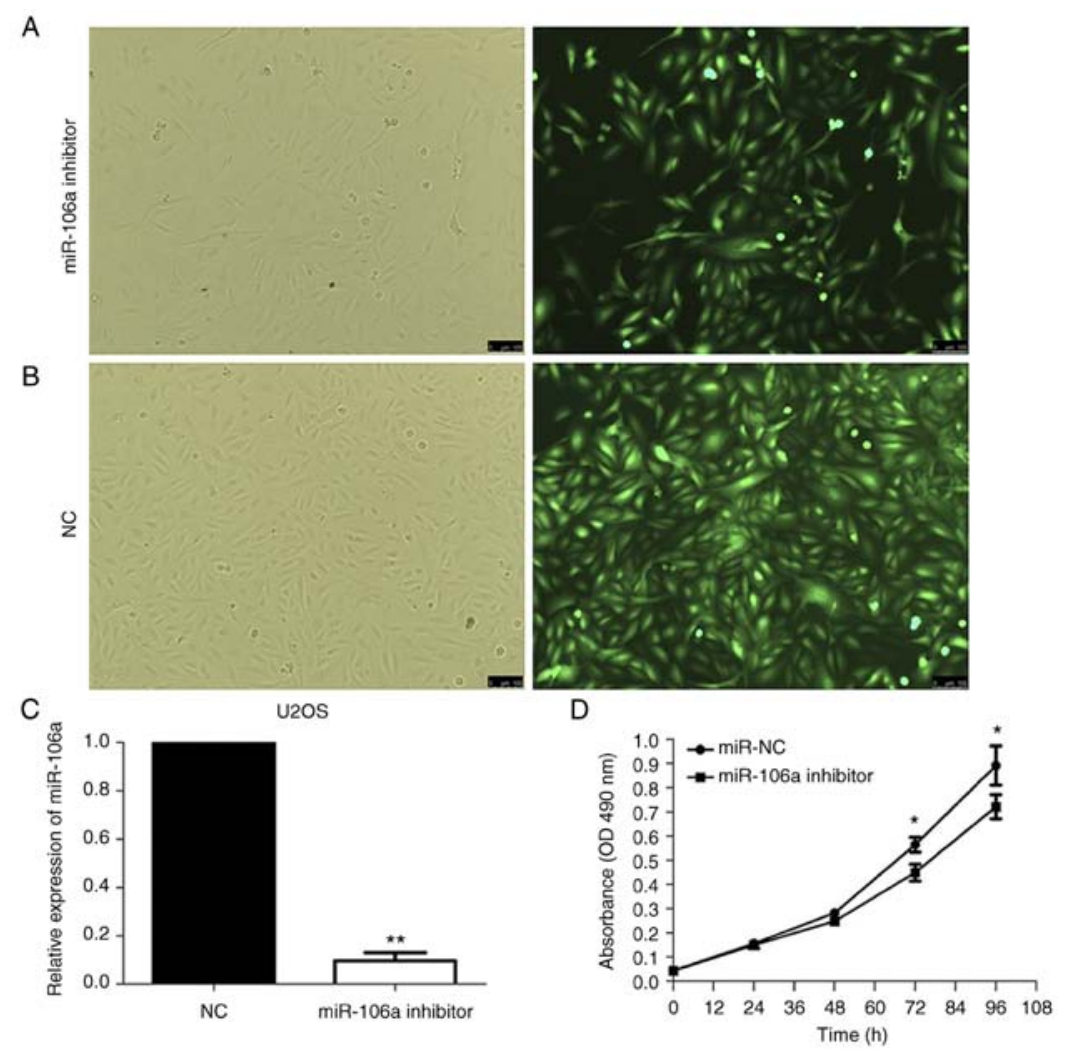

$E$
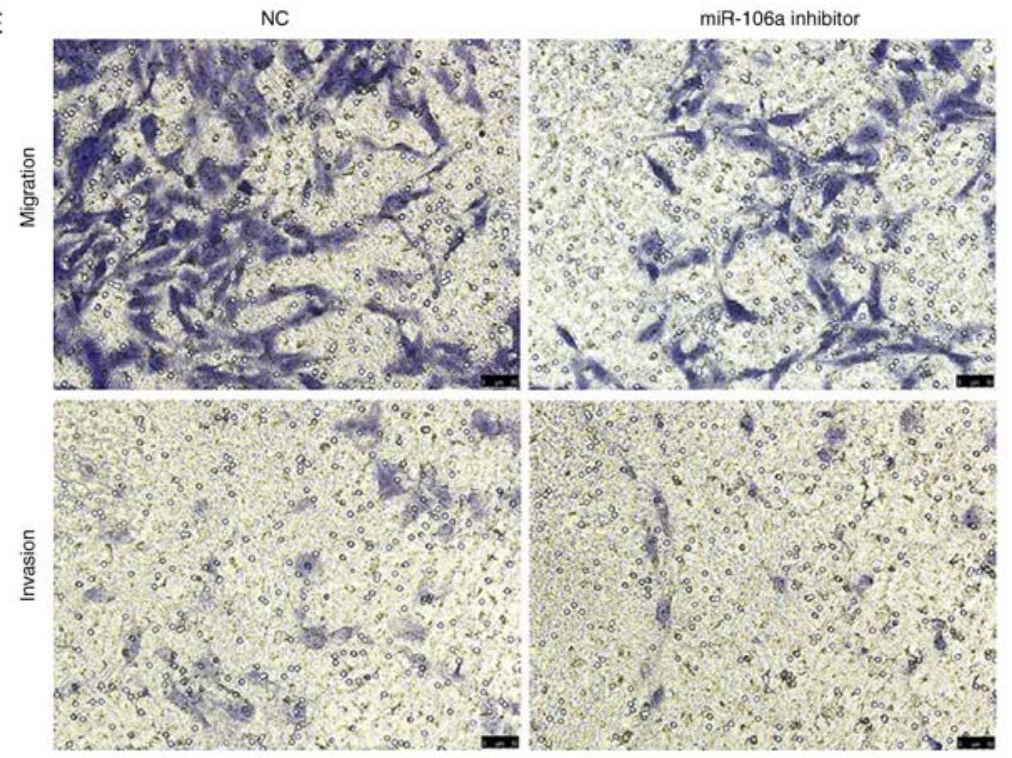

$\mathrm{F}$
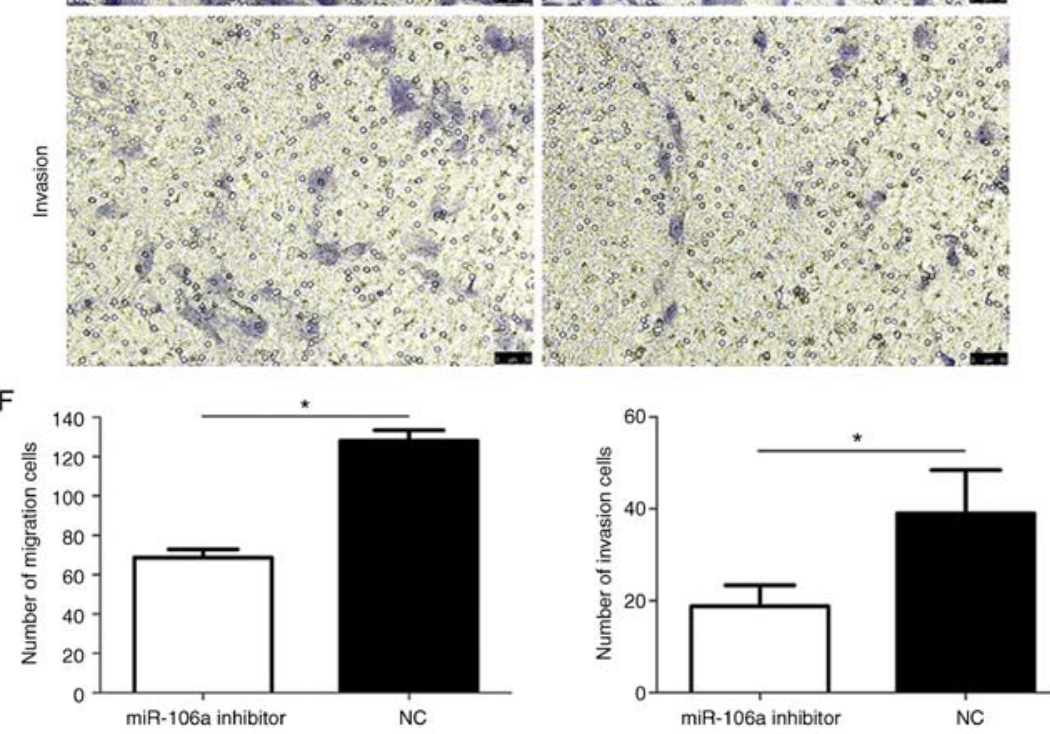

Figure 2. Relative expression of miR-106a in U2OS cells following infection with lentivirus-mediated small interfering RNA and the effects of miR-106a-inhibitor on U2OS cells. The infection efficiency was determined in U2OS cells infected with (A) miR-106a-inhibitor and (B) miR-NC at $72 \mathrm{~h}$ following incubation with lenti-virus at a multiplicity of infection of 10. Bright and green fluorescent view in the same field were observed under a fluorescent microscope. Magnification, x200. Scale bar, $100 \mu \mathrm{m}$. (C) Relative expression of miR-106a in U2OS cells following infection was determined by reverse transcription-quantitative polymerase chain reaction. The expression of miR-106a was normalized to U6. (D) The MTS assay demonstrates the proliferation rate of U2OS cells infected with miR-106a-inhibitor and miR-NC at each time point. (E) Transwell assays indicating the migration and invasion rates of U2OS cells infected with miR-106a-inhibitor and miR-NC. Magnification, $\mathrm{x} 400$. Scale bar, $50 \mu \mathrm{m}$. (F) Quantification of cells that had attached to the surface of the lower chamber. ${ }^{*} \mathrm{P}<0.05,{ }^{* *} \mathrm{P}<0.01$. Data are presented as the mean \pm standard deviation of three independent experiments. miR, microRNA; NC, negative control; OD, optical density. 

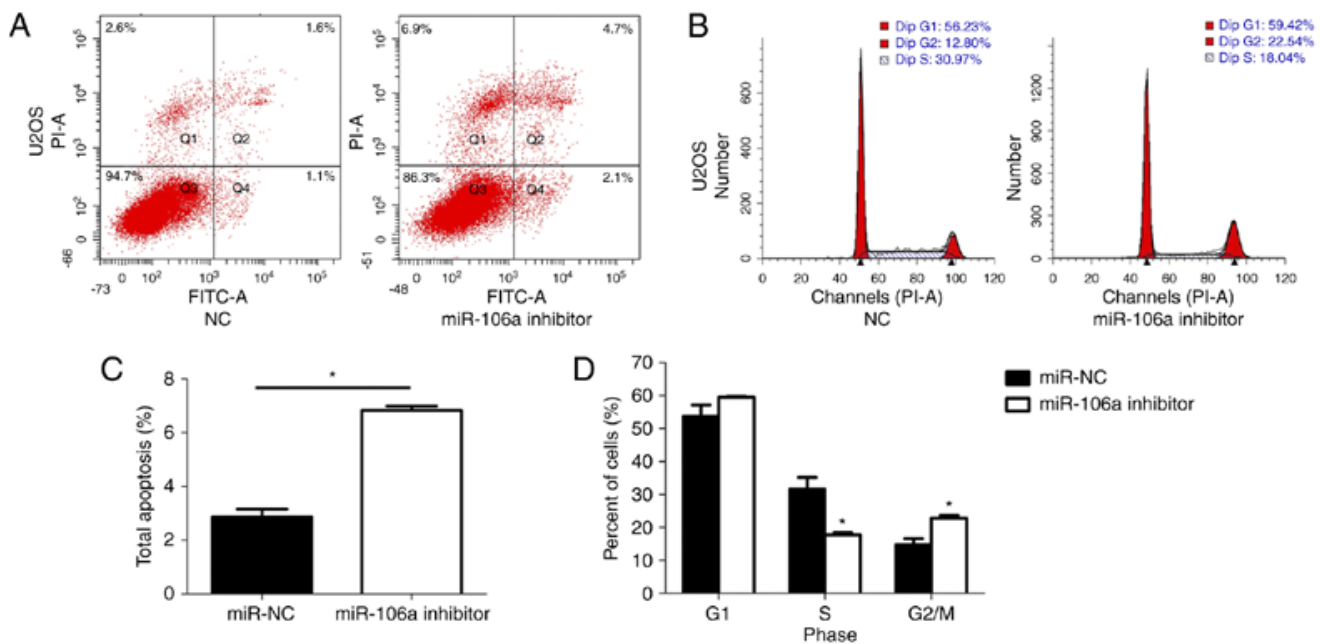

Figure 3. Flow cytometric analysis of apoptosis and cell cycle in U2OS cells infected with miR-106a-inhibitor and miR-NC. (A) Analysis of apoptosis indicating the percentage of total apoptotic cells infected with miR-106a-inhibition and miR-NC. (B) Analysis of cell cycle indicating the percentage of each phase of U2OS cells infected with miR-106a-inhibition and miR-NC. Quantification of (C) apoptosis and (D) cell cycle progression. *P<0.05. Data are presented as the mean \pm standard deviation of three independent experiments. miR, microRNA; NC, negative control.

Scheffe post hoc test was used when more than two groups were present. The spearman's rank correlation test was used to measure the correlation between the expression of miR-106a and VNN2. $\mathrm{P}<0.05$ was considered to indicate a statistically significant difference.

\section{Results}

miR-106a is overexpressed in human osteosarcoma cell lines and patient tissues. To quantify the expression of miR-106a in 18 osteosarcoma and non-cancerous adjacent tissues samples, RT-qPCR was performed. The results indicated that the miR-106a expression level was significantly increased in osteosarcoma tissues compared with NTAs (Fig. 1A). In the human U2OS, Saos-2 and MG63 cells, levels of miR-106a were significantly upregulated, compared with that in normal hFOB1.19 osteoblast cells (Fig. 1B). Since U2OS cells exhibited the highest miR-106a expression level among the three osteosarcoma cell lines, this cell line was chosen for further studies.

Decreased miR-106a expression in human U2OS cells affects cell proliferation and invasion. To explore the association of miR-106a expression with human U2OS cells, the cells were infected with lentivirus-mediated small interfering RNA (miR-106a-inhibitor). Infected cells labeled with green fluorescent protein were observed under a fluorescent microscope, and the infection efficiency was $>90 \%$ when comparing the number of cells using the green fluorescent view in the same field (Fig. 2A and B). Infection efficiency was also determined by RT-qPCR at $72 \mathrm{~h}$ after infection (Fig. 2C).

The MTS assay used to investigate human U2OS cell proliferation following infection with miR-106a-inhibitor or $\mathrm{NC}$ was then performed. The MTS assay was performed at 24, 48, 72 and $96 \mathrm{~h}$. No significant differences were identified in absorbance at $490 \mathrm{~nm}$ in the first $48 \mathrm{~h}$. At 72 and $96 \mathrm{~h}$, a significant decrease in the absorbance at $490 \mathrm{~nm}$ was identified in human U2OS cells infected with miR-106a-inhibitor compared with the NC-infected cells ( $\mathrm{P}<0.05$; Fig. 2D). This indicated that the knockdown of miR-106a expression may have suppressed cell proliferation in human U2OS cells.

Next, an invasion assay was performed to investigate the influence of knockdown of miR-106a of human U2OS cells on the migration and invasion ability. Cells that passed through the upper chamber and attached to the lower surface of the membrane were fixed (Fig. 2E) and quantified (Fig. 2F). The number of miR-106a-inhibitor-infected U2OS cells that passed through the upper chamber was significantly decreased compared with that of miR-NC-infected cells.

Decreased miR-106a expression in human U2OS cells influences the cell apoptosis ratio and cycle distribution. In order to explore the mechanisms underlying the inhibitory effect of miR-106a-inhibitor infection on cell proliferation and invasion, flow cytometric analysis of apoptosis and the cell cycle were applied (Fig. 3). As the Annexin V and PI staining results demonstrated, knockdown of miR-106a resulted in a significant increase in the apoptosis rate of human U2OS cells from 2.7 to $6.7 \%$ compared with miR-NC-infected cells $(\mathrm{P}<0.05$; Fig. 3A). The cell cycle analysis demonstrated that knockdown of miR-106a significantly increased the percentage of cells in the $\mathrm{G} 2 / \mathrm{M}$ phase from 12.80 to $22.54 \%$ and decreased the percentage in the $\mathrm{S}$ phase from 30.97 to $18.04 \%$ (both $\mathrm{P}<0.05$; Fig. 3B).

Knockdown of miR-106a expression in human U2OS cells leads to an increase in VNN2 expression. To explore whether VNN2 is a candidate target gene of miR-106a, VNN2 protein expression levels were detected by western blot analysis in human U2OS cells following the knockdown of miR-106a (Fig. 2C). The results revealed a significant upregulation in VNN2 protein expression in knocked down U2OS cells compared with miR-NC-infected cells (Fig. 4A).

RT-qPCR was performed to analyze the effect of downregulating miR-106a expression on VNN2 expression at the transcriptional level. The expression level of VNN2 mRNA was 
A

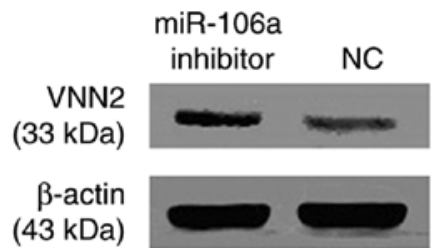

B

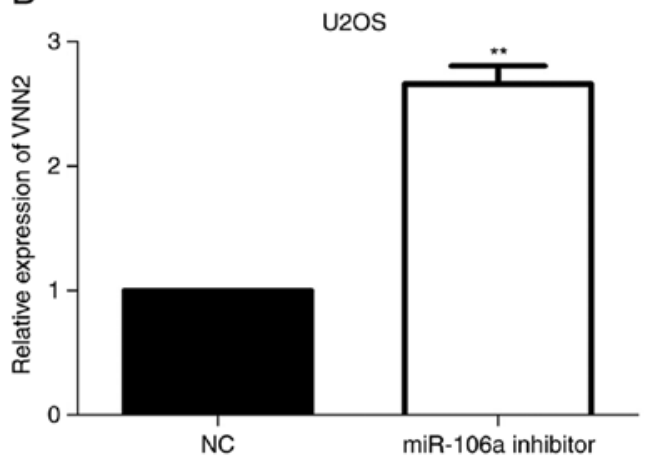

Figure 4. Relative expression of VNN2 in U2OS cells following knockdown of miR-106a. (A) Western blot analysis indicating VNN2 expression at protein level in U2OS cells at $72 \mathrm{~h}$ postinfection with miR-106a-inhibitor or miR-NC. (B) Reverse transcription-quantitative polymerase chain reaction indicating VNN2 expression at mRNA level in U2OS cells at $72 \mathrm{~h}$ postinfection with miR-106a-inhibitor or miR-NC. Expression of VNN2 mRNA was normalized to GAPDH. ${ }^{* *} \mathrm{P}<0.01$. Data are presented as the mean \pm standard deviation of three independent experiments. miR, microRNA; NC, negative control; VNN2, vascular non-inflammatory molecule 2 .

significantly increased in miR-106a inhibitor-infected U2OS cells compared with that in $\mathrm{NC}$-infected U2OS cells $(\mathrm{P}<0.05$; Fig. 4B). An inverse association between miR-106a expression and VNN2 expression levels was verified via RT-qPCR and western blot analysis.

miR-106a directly targets VNN2. By using open access online databases (TargetScan, PicTarget and miRBase Targets), VNN2 was initially identified as a candidate target gene of miR-106a. A complementary site of miR-106a was identified in the VNN2 3'-UTR (Fig. 5A).

To confirm the possibility that miR-106a directly targets VNN2, the miR-106a binding region at the 3'-UTR of VNN2 or VNN2-mut mRNA were cloned downstream of the firefly luciferase reporter gene in GV272 vectors. miR-106a or miR-NC vectors and GV272 vectors were then co-transfected into $293 \mathrm{~T}$ cells. The relative luciferase activity of the reporter containing VNN2 3'-UTR was significantly suppressed by $54 \pm 0.03 \%(\mathrm{P}<0.05)$ when miR-106a vectors were co-transfected, whereas the relative luciferase activity of 3'-UTR-NC reporter was not affected (Fig. 5B). The results of luciferase activity assay indicated that miR-106a may target and suppress VNN2 gene expression by binding the 3'-UTR of VNN2 mRNA.

$V N N 2$ expression level is inversely correlated with expression of miR-106a in human osteosarcoma tissues. To quantify the expression of VNN2 in 18 osteosarcoma tissue and NTA samples, RT-qPCR was performed. The results indicated that the VNN2 expression level was significantly decreased in osteosarcoma tissues, compared with NTAs (Fig. 6A). Finally, the spearman's rank correlation test was used to analyze the expression of VNN2 and miR-106a in 18 osteosarcoma with non-cancerous adjacent tissues. The result indicated a negative correlation between them $(\mathrm{R}=-0.640, \mathrm{P}<0.01$; Fig. $6 \mathrm{~B})$.
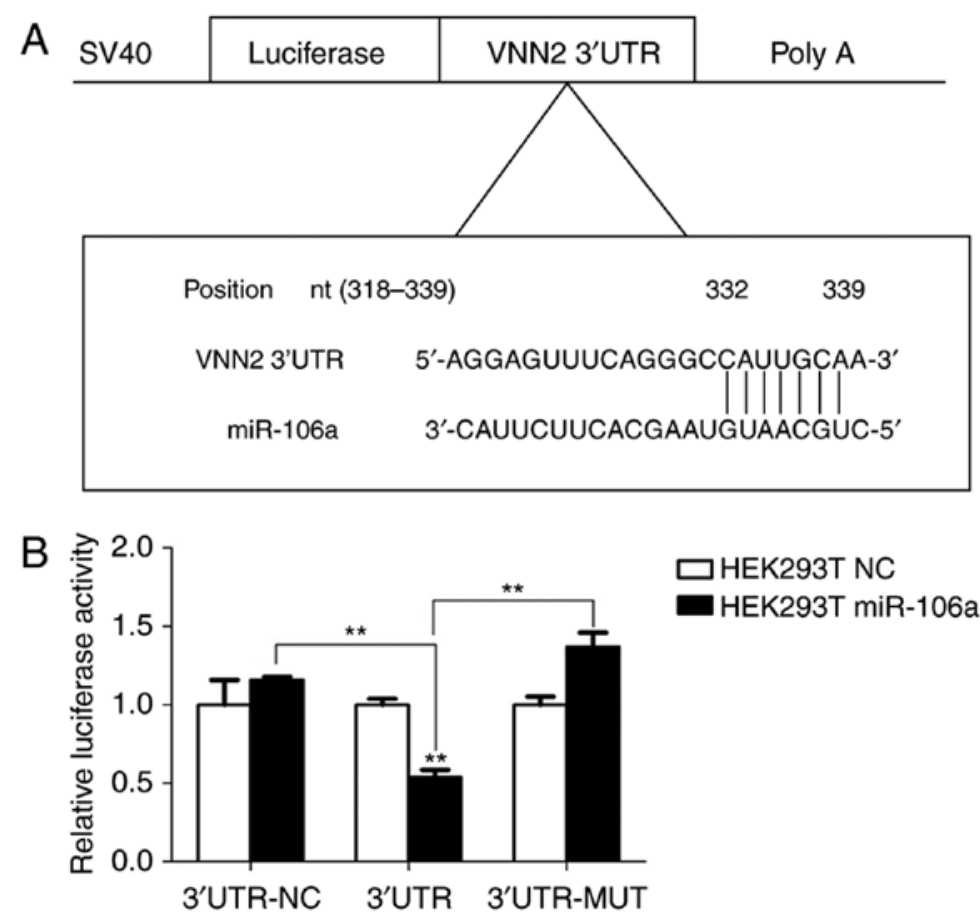

Figure 5. VNN2 is a direct target of miR-106a. (A) Bioinformatic analysis indicating the prediction of the complementary site of miR-106a in the VNN2 3'-UTR. (B) Co-transfection of VNN2 3'-UTR and miR-106a plasmid vectors in 293T cells, the relative luciferase activity are presented. ${ }^{* *}$ P $<0.01$. Data are presented as the mean \pm standard deviation of three independent experiments. miR, microRNA; 3'-UTR, 3'untranslated region; VNN2, vascular non-inflammatory molecule 2 ; NC, negative control; Mut, mutant. 

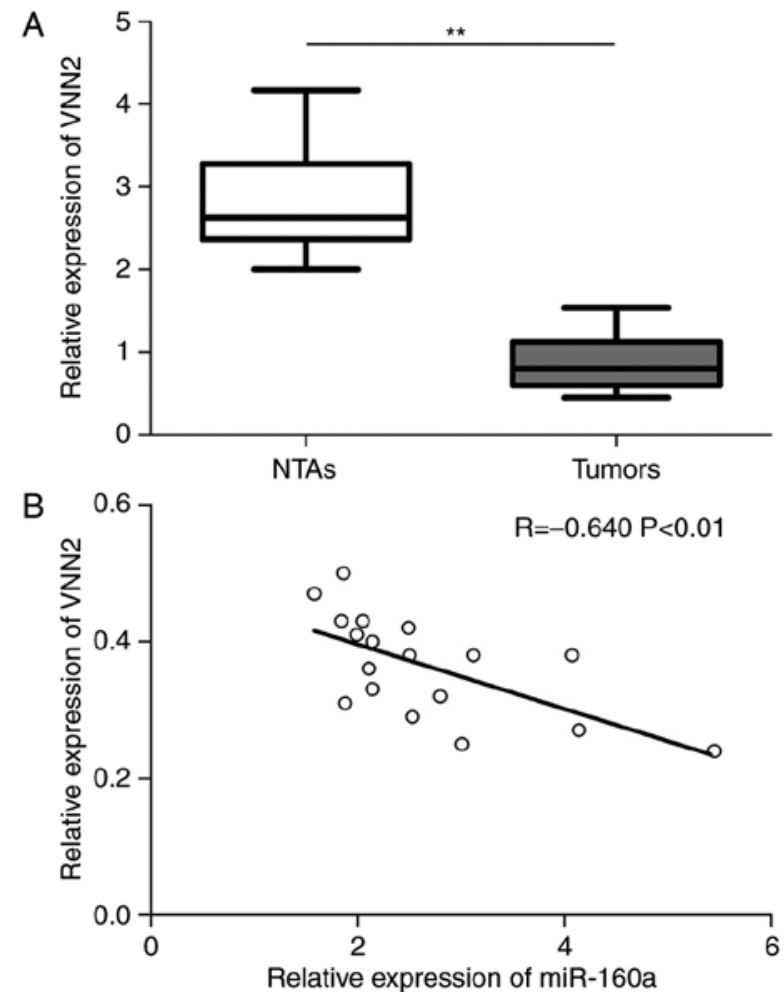

Figure 6. Correlation between VNN2 and miR-106a in osteosarcoma tissues and NTAs. (A) Relative expression of VNN2 in 18 osteosarcoma samples compared with NTAs as determined by reverse transcription-quantitative polymerase chain reaction. ${ }^{* *} \mathrm{P}<0.01$. (B) An inverse correlation was identified between levels of miR-106a and VNN2 in 18 osteosarcoma tissues and NTAs. ${ }^{* *} \mathrm{P}<0.01$. The expression of miR-106a and VNN2 mRNA was normalized to U6 and GAPDH, respectively. The spearman's rank correlation test was used to measure the degree of correlation between variables, and Spearman's rho and P-values (two-tailed) are presented. Data are presented as the mean \pm standard deviation of three independent experiments. miR, microRNA; NTAs, non-cancerous adjacent tissues.

\section{Discussion}

MicroRNAs emerged as novel gene regulators and have been extensively studied in various types of human cancer $(24,25)$. Up to a third of the protein-coding genes in the human genome are regulated by $>2,000$ microRNAs that have been discovered in human so far (26). The function of microRNA is primarily ascribed to the dynamic regulation of human cancer as oncogenes or tumor suppressors (27). Based on previous studies, the same type of microRNA exhibit similar functions in different types of human cancer. For example, microRNA-21 has been detected to be significantly upregulated in liver cancer, breast cancer and malignant glioma $(11,28,29)$. MicroRNA-21 is able to promote the proliferation and invasion of hepatocellular carcinoma cells by downregulating the expression of tumor suppressor phosphatase and tensin homolog, while the knockdown of microRNA-21 in malignant glioma results in caspase activation, inducing an increase in apoptosis (29). It has also been reported that the tumorigenic ability of the microRNA-21-knockdown MCF-7 breast cancer cell line is significantly reduced in nude mice, and further studies have demonstrated that microRNA-21 may promote cell proliferation by inhibiting the target gene tropomyosin 1 (30). Furthermore, the upregulation of miR-21 indicates a positive correlation with advanced clinicopathological features and poor prognosis in patients of renal cell carcinoma (31). These studies indicate the proto-oncogene activity of microRNA-21 and suggest that the same microRNA in different types of human cancer has similar functions.

Recent several studies have reported that microRNA-106a is frequently upregulated in different types of human cancer, and is involved in tumor development, initiation, progression, invasion and metastasis $(32,33)$. Shen et al (34) demonstrated that the expression of microRNA-106a was increased in thyroid cancer, and miRNA-106a directly targeting retinoic acid receptor $\beta$ was associated with the viability, apoptosis, differentiation and the iodine uptake function of thyroid cancer cell lines by regulating mitogen activated protein kinase signaling pathway in vitro. Espinosa-Parrilla et al (35) identified that genetic variation in microRNA-106a had an essential role in genetic susceptibility to gastric cancer and contributed to the molecular mechanisms of gastric carcinogenesis. These results demonstrated the important role of microRNA-106a in liver and gastric cancer. However, there is limited data available regarding the functional role and underlying mechanism of microRNA-106a in osteosarcoma. Therefore, the aim of the present study was to detect the expression level and to explore the possible target of microRNA-106a in osteosarcoma.

In the present study, it was revealed that microRNA-106a was significantly upregulated in osteosarcoma tissue compared with the adjacent normal tissue as well as in osteosarcoma cell lines, which was contrary to another study whereby miR-106a-5p was downregulated in osteosarcoma tissues (36). In the present study, knockdown of microRNA-106a significantly the inhibited malignant phenotypic processes of osteosarcoma, including proliferation, migration and invasion. Cell cycle and apoptosis analysis were subsequently applied to reveal the mechanisms underlying these phenotypic changes. The cytometric results demonstrated that the knockdown of microRNA-106a resulted in significantly increased apoptosis rates, decreased the percentage of cells in the $S$ phase and increased the cell population in the $\mathrm{G} 2 / \mathrm{M}$ phase. To determine the potential target of microRNA-106a, online databases were used, which identified VNN2 as a candidate gene. This result supports the hypothesis that microRNA-106a acts as an oncogene by targeting VNN2. To verify this hypothesis and the prediction of online databases, RT-qPCR and western blotting were performed to determine the expression of VNN2 in U2OS cells following knockdown of microRNA-106a compared with NC. There was reasonable concordance whereby the expression of VNN2 was significantly upregulated in both cases. Additional, the expression of VNN2 in osteosarcoma tissue compared with the adjacent normal tissue was detected by RT-qPCR. It was demonstrated that the VNN2 expression level was significantly decreased in osteosarcoma tissues compared with NTAs, and an inverse correlation between miR-106a and VNN2 expression was observed. Additionally, dual-luciferase was performed to further confirm the possibility that microRNA-106a targets VNN2. The relative luciferase activity of VNN2 3'-UTR group was significantly suppressed, which indicated that microRNA-106a was able to bind to the 3'-UTR of VNN2 mRNA, thereby affecting proliferation, invasion and apoptosis in osteosarcoma. 
It is generally known that oxidative phosphorylation is replaced by aerobic glycolysis in tumor cells, which only makes use of metabolic recombination to maintain intracellular ATP and NADH levels instead of obtaining ATP via mitochondria (37). An enormous amount of ATP and NADH are necessary for tumor cell proliferation and metastasis, and also to maintain the base metabolism of tumor cells. Destruction of tumor cell energy and redox substances may be associated with the molecular mechanisms that underlie metabolic reprogramming of cancer cells (38). VNN2 as a pantetheine hydrolase serves an important role in the pantothenate and coenzyme A biosynthesis pathway (39). Considering the cell cycle dysregulation and apoptosis rate changes observed in the present study, we hypothesized that VNN2 regulates the aerobic glycolysis of tumor cells and alter metabolite-driven gene regulation of osteosarcoma cells to induce apoptosis and cell cycle changes. Verification of this hypothesis is planned in future studies.

In conclusion, the overexpression of microRNA-106a in human osteosarcoma tissues and U2OS cell line was observed, and the knockdown of microRNA-106a was demonstrated to inhibit osteosarcoma cell proliferation and invasion and induce apoptosis by targeting VNN2. The current study identified microRNA-106a may be a potential novel diagnostic marker and targeting microRNA-106a-VNN2 interaction may be a potential therapeutic strategy in human osteosarcoma. However, the function of VNN2 in osteosarcoma remains unclear, and further studies are required to identify the underlying mechanisms of VNN2 effects on osteosarcoma cells.

\section{Acknowledgements}

Not applicable.

\section{Funding}

The present study was supported by the Natural Science Foundation of Science and Technology Department of Liaoning Province (grant no. 201302106) and the Science and Technology Department of Shenyang City (grant no. F14-231-1-48).

\section{Availability of data and materials}

All data generated or analyzed during this study are included in this published article.

\section{Authors' contributions}

HT conceived and designed the experiments. CY, YX and LC performed the experiments. CY, LC and ZS wrote the manuscript. CY, LP, WZ and ZS performed the data analysis. All authors read and approved the manuscript.

\section{Ethics approval and consent to participate}

The present study received approval by the Ethics Committee of the First Affiliated Hospital of China Medical University (Shenyang, China) and written informed consent was obtained from all patients.

\section{Patient consent for publication}

Not applicable.

\section{Competing interests}

The authors declare that they have no competing interests.

\section{References}

1. Ward E, DeSantis C, Robbins A, Kohler B and Jemal A: Childhood and adolescent cancer statistics, 2014. CA Cancer J Clin 64: 83-103, 2014.

2. Mirabello L, Troisi RJ and Savage SA: Osteosarcoma incidence and survival rates from 1973 to 2004: Data from the Surveillance, Epidemiology, and End Results program. Cancer 115: 1531-1543, 2009.

3. Bielack SS, Kempf-Bielack B, Delling G, Exner GU, Flege S, Helmke K, Kotz R, Salzer-Kuntschik M, Werner M, Winkelmann W, et al: Prognostic factors in high-grade osteosarcoma of the extremities or trunk: An analysis of 1,702 patients treated on neoadjuvant cooperative osteosarcoma study group protocols. J Clin Oncol 20: 776-790, 2002.

4. Chou AJ, Kleinerman ES, Krailo MD, Chen Z, Betcher DL, Healey JH, Conrad EU III, Nieder ML, Weiner MA, Wells RJ, et al: Addition of muramyl tripeptide to chemotherapy for patients with newly diagnosed metastatic osteosarcoma: A report from the Children's Oncology Group. Cancer 115: 5339-5348, 2009.

5. Aung L, Tin AS, Quah TC and Pho RW: Osteogenic sarcoma in children and young adults. Ann Acad Med Singapore 43: 305-313, 2014.

6. Behm-Ansmant I, Rehwinkel J and Izaurralde E: MicroRNAs silence gene expression by repressing protein expression and/or by promoting mRNA decay. Cold Spring Harb Symp Quant Biol 71: 523-530, 2006.

7. Ambros V: MicroRNA pathways in flies and worms: Growth, death, fat, stress, and timing. Cell 113: 673-676, 2003.

8. Song L and Tuan RS: MicroRNAs and cell differentiation in mammalian development. Birth Defects Res C Embryo Today 78: 140-149, 2006

9. Skaftnesmo KO, Prestegarden L, Micklem DR and Lorens JB: MicroRNAs in tumorigenesis. Curr Pharm Biotechnol 8: 320-325, 2007.

10. Lulla RR, Costa FF, Bischof JM, Chou PM, de F Bonaldo M, Vanin EF and Soares MB: Identification of differentially expressed MicroRNAs in osteosarcoma. Sarcoma 2011: 732690, 2011.

11. Meng F, Henson R, Wehbe-Janek H, Ghoshal K, Jacob ST and Patel T: MicroRNA-21 regulates expression of the PTEN tumor suppressor gene in human hepatocellular cancer. Gastroenterology 133: 647-658, 2007.

12. He L, He X, Lim LP, de Stanchina E, Xuan Z, Liang Y, Xue W, Zender L, Magnus J, Ridzon D, et al: A microRNA component of the p53 tumour suppressor network. Nature 447: 1130-1134, 2007.

13. Cimmino A, Calin GA, Fabbri M, Iorio MV, Ferracin M, Shimizu M, Wojcik SE, Aqeilan RI, Zupo S, Dono M, et al: $m i R-15$ and $m i R-16$ induce apoptosis by targeting BCL2. Proc Natl Acad Sci USA 102: 13944-13949, 2005.

14. Palmini G, Marini F and Brandi ML: What is new in the miRNAWorld regarding osteosarcoma and chondrosarcoma? Molecule 22: pii: E417, 2017.

15. Huang J, Takeda Y, Watanabe T and Sendo F: A sandwich ELISA for detection of soluble GPI-80, a glycosylphosphatidyl-inositol (GPI)-anchored protein on human leukocytes involved in regulation of neutrophil adherence and migration-its release from activated neutrophils and presence in synovial fluid of rheumatoid arthritis patients. Microbiol Immunol 45: 467-471, 2001.

16. Suzuki K, Watanabe T, Sakurai S, Ohtake K, Kinoshita T, Araki A, Fujita T, Takei H, Takeda Y, Sato Y, et al: A novel glycosylphosphatidyl inositol-anchored protein on human leukocytes: A possible role for regulation of neutrophil adherence and migration. J Immunol 162: 4277-4284, 1999. 
17. Singel KL and Segal BH: Neutrophils in the tumor microenvironment: Trying to heal the wound that cannot heal. Immunol Rev 273: 329-343, 2016.

18. Eruslanov EB, Bhojnagarwala PS, Quatromoni JG, Stephen TL, Ranganathan A, Deshpande C, Akimova T, Vachani A, Litzky L, Hancock WW, et al: Tumor-associated neutrophils stimulate $\mathrm{T}$ cell responses in early-stage human lung cancer. J Clin Invest 124: 5466-5480, 2014

19. Nitto T, Araki Y, Takeda Y and Sendo F: Pharmacological analysis for mechanisms of GPI-80 release from tumour necrosis factor-alpha-stimulated human neutrophils. Br J Pharmacol 137: 353-360, 2002

20. Li P, Liu H, Zhang Z, Lv X, Wang H, Ma J, Ma Z, Qu X and Teng YE: Expression and comparison of Cbl-b in lung squamous cell carcinoma and adenocarcinoma. Med Sci Monit 24: 623-635, 2018.

21. Livak KJ and Schmittgen TD: Analysis of relative gene expression data using real-time quantitative PCR and the $2^{-\Delta \Delta C_{\mathrm{T}}}$ method. Methods 25: 402-408, 2001.

22. Justus CR, Leffler N, Ruiz-Echevarria M and Yang LV: In vitro cell migration and invasion assays. J Vis Exp: Jun 1, 2014. doi: $10.3791 / 51046$.

23. Mahmood T and Yang PC: Western blot: Technique, theory, and trouble shooting. N Am J Med Sci 4: 429-434, 2012.

24. Gregory PA, Bert AG, Paterson EL, Barry SC, Tsykin A, Farshid G, Vadas MA, Khew-Goodall Y and Goodall GJ: The miR-200 family and miR-205 regulate epithelial to mesenchymal transition by targeting ZEB1 and SIP1. Nat Cell Biol 10: 593-601, 2008.

25. Johnson SM, Grosshans H, Shingara J, Byrom M, Jarvis R, Cheng A, Labourier E, Reinert KL, Brown D and Slack FJ: RAS is regulated by the let-7 microRNA family. Cell 120: 635-647, 2005 .

26. Hammond SM: An overview of microRNAs. Adv Drug Deliv Rev 87: 3-14, 2015.

27. Calin GA and Croce CM: MicroRNA signatures in human cancers. Nat Rev Cancer 6: 857-866, 2006.

28. Iorio MV, Ferracin M, Liu CG, Veronese A, Spizzo R, Sabbioni S, Magri E, Pedriali M, Fabbri M, Campiglio M, et al: MicroRNA gene expression deregulation in human breast cancer. Cancer Res 65: 7065-7070, 2005
29. Chan JA, Krichevsky AM and Kosik KS: MicroRNA-21 is an antiapoptotic factor in human glioblastoma cells. Cancer Res 65: 6029-6033, 2005.

30. Zhu S, Si ML, Wu H and Mo YY: MicroRNA-21 targets the tumor suppressor gene tropomyosin 1 (TPM1). J Biol Chem 282: 14328-14336, 2007.

31. Liu Z, Lu Y, Xiao Y and Lu Y: Upregulation of miR-21 expression is a valuable predicator of advanced clinicopathological features and poor prognosis in patients with renal cell carcinoma through the p53/p21-cyclin E2-Bax/caspase-3 signaling pathway. Oncol Rep 37: 1437-1444, 2017.

32. Edatt L, Maurya AK, Raji G, Kunhiraman H and Kumar SV: MicroRNA106a regulates matrix metalloprotease 9 in a sirtuin-1 dependent mechanism. J Cell Physiol 233: 238-248, 2018.

33. Pan YJ, Zhuang Y, Zheng JN and Pei DS: MiR-106a: Promising biomarker for cancer. Bioorg Med Chem Lett 26: 5373-5377, 2016.

34. Shen CT, Qiu ZL, Song HJ, Wei WJ and Luo QY: miRNA-106a directly targeting RARB associates with the expression of $\mathrm{Na}^{+} / \mathrm{I}$ symporter in thyroid cancer by regulating MAPK signaling pathway. J Exp Clin Cancer Res 35: 101, 2016.

35. Espinosa-Parrilla Y, Munoz X, Bonet C, Garcia N, Venceslá A, Yiannakouris N, Naccarati A, Sieri S, Panico S, Huerta JM, et al: Genetic association of gastric cancer with miRNA clusters including the cancer-related genes MIR29, MIR25, MIR93 and MIR106: Results from the EPIC-EURGAST study. Int J Cancer 135: 2065-2076, 2014.

36. He QY, Wang GC, Zhang H, Tong DK, Ding C, Liu K, Ji F, Zhu X and Yang $\mathrm{S}$ : miR-106a-5p suppresses the proliferation, migration, and invasion of osteosarcoma cells by targeting $H M G A 2$. DNA Cell Biol 35: 506-520, 2016.

37. Kroemer $\mathrm{G}$ and Pouyssegur J: Tumor cell metabolism: Cancer's Achilles' heel. Cancer Cell 13: 472-482, 2008.

38. Lopez-Rios F, Sanchez-Arago M, Garcia-Garcia E, Ortega AD, Berrendero JR, Pozo-Rodríguez F, López-Encuentra A, Ballestín C and Cuezva JM: Loss of the mitochondrial bioenergetic capacity underlies the glucose avidity of carcinomas. Cancer Res 67: 9013-9017, 2007.

39. Nitto T and Onodera K: Linkage between coenzyme a metabolism and inflammation: Roles of pantetheinase. J Pharmacol Sci 123: $1-8,2013$ 